\title{
The IART System for Race Walking: Experience with World-Class Olympic Race Walkers ${ }^{\dagger}$
}

\author{
Teodorico Caporaso ${ }^{1, *}$, Stanislao Grazioso ${ }^{1}$, Dario Panariello ${ }^{1,2}$, Giuseppe Di Gironimo ${ }^{1}$ \\ and Antonio Lanzotti ${ }^{1}$ \\ 1 Fraunhofer JL IDEAS, Department of Industrial Engineering, University of Naples Federico II, \\ 80125 Naples, Italy; stanislao.grazioso@unina.it (S.G.); dario.panariello@unibg.it (D.P.); \\ giuseppe.digironimo@unina.it (G.D.G.); antonio.lanzotti@unina.it (A.L.) \\ 2 Department of Management, Information and Production Engineering, University of Bergamo, \\ 24044 Bergamo, Italy \\ * Correspondence: teodorico.caporaso@unina.it \\ + Presented at the 13th conference of the International Sports Engineering Association, Online, \\ 22-26 June 2020.
}

Published: 15 June 2020

\begin{abstract}
Race walking is a discipline in which the best chronometric performance is constrained by infringements. Currently, the judgment and training of race walkers is entrusted to subjective observations made by judges. In this paper, with the objective of supporting coaching and judging, we present a wearable inertial sensor system (IART) for the evaluation of performances and infringements in race walking. The system is composed of an inertial sensor positioned close to the center of mass of the subject and a management unit designed for coaches and judges. IART allows: (i) a step sequence classification according to the competition rules; (ii) a customized assessment of elite race walkers based on key performance and infringement indices. The system is experimentally validated in field conditions by nine world-class Olympic race walkers. The results show that IART improves the current evaluation of step sequences and offers a meaningful support for the overall evaluation of the technical gesture.
\end{abstract}

Keywords: race walking; wearable inertial sensor; training; judgments; biomechanics; elite athlete

\section{Introduction}

The recent history shows that, at various levels and in many disciplines, the technological evolution has radically changed the way the sport is approached from the point of view of monitoring, judging, and training. In this work we focus on technological innovation in race walking.

In race walking, according to rule 230 of the IAAF competition rules [1], the athlete could occur in two possible infringements: "bent knee" and "loss of ground contact" (LOGC). For elite race walkers, the most common infringement is LOGC, as resulting from the last main international events [2]. It is worth noticing that nowadays judges and coaches can rely only on their subjective observations (made by human eyes); indeed, to date, technology is not used to support judging decisions. By relying mainly on subjective observations, there is a critical issue during race walking competitions: the very short duration of the loss of ground contact events (few hundredths of a second) generates difficulties for the proper identification of a correct/incorrect gesture [3].

On the other hand, performance optimization might determine a good or bad final result. For example, increasing the step length (SL) even of a single centimeter can lead to a time improvement of about $2 \mathrm{~min}$ at the end of $50 \mathrm{~km}$ : to have an idea, this duration is greater than the time duration between the first and the fourth at the last Olympic games [4]. Although in elite race walkers the 
importance of SL on race walking speed appears greater than step cadence (SC), it should be noticed that there is a limit on how much SL can be increased before achieving dangerous SC values. Furthermore, increases in SL might be achieved through longer LOGC. As a matter of fact, the ability of the best race walkers consists in achieving the optimal SL and SC (with a legal LOGC) [5].

In order to support judgement and training in field conditions (the real competitive and training scenario) two possible technologies are available: insole pressure and inertial systems. The most recent evidence in the literature [6,7] underlines how wearable inertial sensors, located in different positions on the human body, could be a suitable technology to provide quantitative and reliable data useful for measuring and monitoring an athlete's performance and technique. In this context, the Inertial Assistant Referee and Trainer (IART) for race-walking system represents an interesting supporting tool. The IART architecture is shown in Figure 1: the measurement unit is placed at the bottom of the race walker's vertebral column, while the management unit is a mobile app designed for the main users (i.e., coaches and judges).

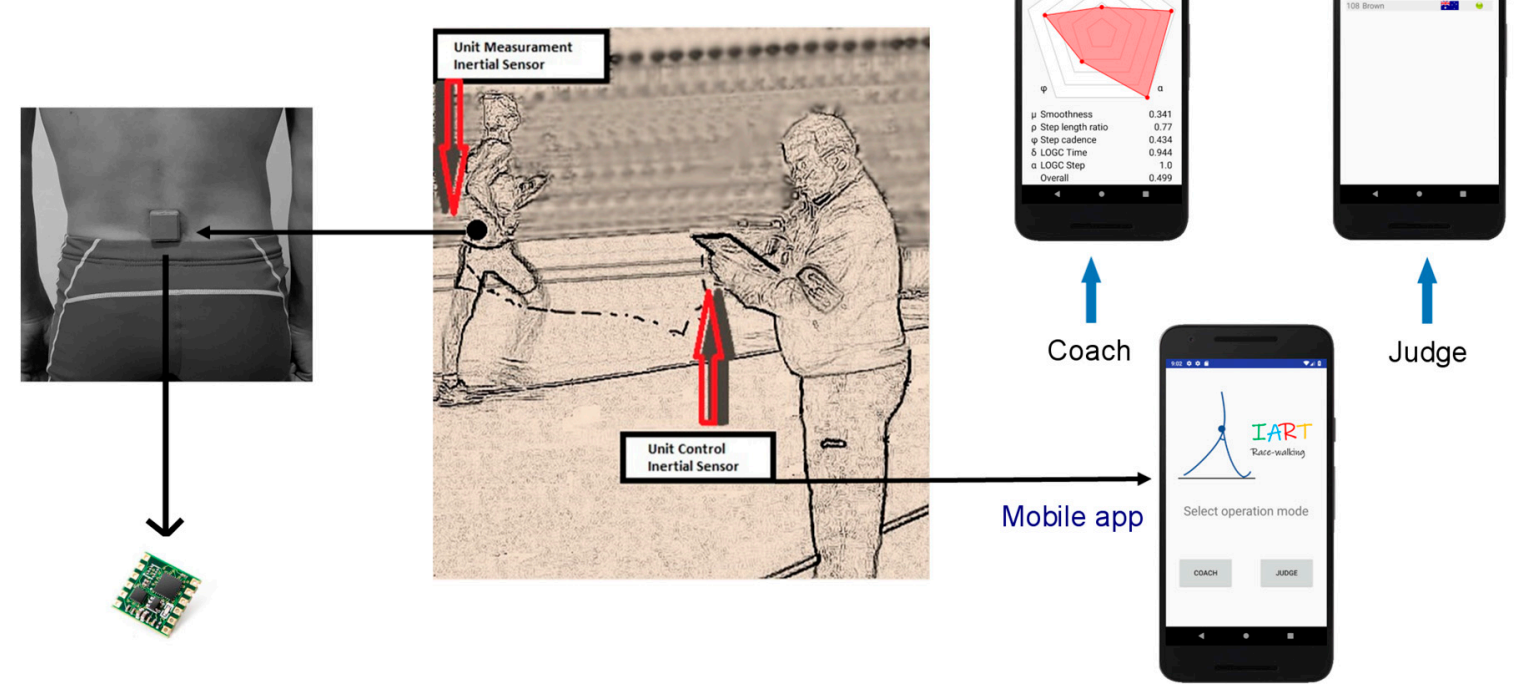

Figure 1. In the center, an example of a real scenario; the measurement unit (displayed on the left) is placed in communication with the management unit. An image of the mobile app (on the right) shows two possible operation modes (judge and coach).

In the following part of this paper, we describe the IART device, composed of measurement and management units. Then, we present the use of the IART system in a real experimental scenario as a tool for the evaluation of performance and infringements. In particular, the experiments involve nine world-class Olympic race walkers.

\section{Materials and Methods}

\subsection{Measurament Unit}

The measurement unit was chosen to satisfy the following functional requirements: (i) sample frequency and dynamic range adapted to achieve a good reliability in relation to the characteristics of the race walking gesture; (ii) small volume and lightweight design (in order to obtain a comfortable product); (iii) rechargeable battery with an operating time at least equal to $4-5 \mathrm{~h}$ (the maximum duration of the longest competition distance, $50 \mathrm{~km}$ ); (iv) availability to transfer the collected data to a management unit [8]. Thus, we choose an inertial system (i.e., the model type G-Sensor2, BTS) with the following technical features able to satisfy the previous functional requirements: (i) Sample Frequency (SF) of $200 \mathrm{~Hz}(1 / 5 \mathrm{~ms})$ and dynamic range $\pm 8 \mathrm{~g}$ for the tri-axis accelerometer and $\pm 300 \mathrm{gps}$ for the tri-axis gyroscope sensor; (ii) weight of $62 \mathrm{~g}$ and dimensions equal to $78 \times 48 \times 20 \mathrm{~mm}$; (iii) 
rechargeable battery with an operating time of about $24 \mathrm{~h}$; (iv) collected data stored on the sensor and then transmitted via Bluetooth to a mobile device.

\subsection{Management Unit}

The management unit has indeed to satisfy the following functional requirement: (i) it has to be user-adjustable, in order to have more reliable output (efficient); (ii) it should have a user-friendly interface (easy to use) [8]. For this aim, we chose a management unit that offers: (i) two-mode operation (customized for coaches and judges); (ii) a simple color-based output for the judges and a graphical output (a radar chart representation) for the coaches.

Figure 2 shows the flowchart of the process implemented in the management unit. The first part of the workflow is related to the judge operation mode. The process starts with the processing of inertial data, which includes the following steps [9]: (i) filtering with low-pass Butterworth filters of raw data regarding vertical and anteroposterior accelerations; (ii) individuation of temporal events ( $t_{\text {max,i: }}$ time related to the maximum in the anteroposterior acceleration related to the generic step $i$ and, $t_{\min , i}$ time related to the maximum in the anteroposterior acceleration related to the generic step i) on the accelerations timeline based on the location of a peak point having a specific value (in relation to $\mathrm{W}$ : fixed temporal value for the assessment of $t_{\text {min,i }}$ and $\mathrm{V}$ : fixed temporal value for the assessment of $t_{\max , i}$ ) ; (iii) assessment of $\mathrm{SC}_{\mathrm{i}}$, (SC related to generic step i) useful for the definition of $\mathrm{E}_{\mathrm{i}}$ (the temporal threshold related to generic step $i$ ) through a quadratic correlation; (iv) LOGC timing $\left(\mathrm{LOGC}_{\mathrm{T}}\right)$ assessment based on $\mathrm{E}_{\mathrm{i}}, \mathrm{t}_{\max , \mathrm{i}}$, and $\mathrm{t}_{\mathrm{min}, \mathrm{i}}$. This part of the flow chart allows to obtain the output required by the judge operation mode. Starting from LOGC $_{\mathrm{T}}$, the mean value of $\mathrm{LOGC}_{\mathrm{T}}$ for a sequence of steps is calculated. Indeed, according to the IAAF regulations, the judges must consider a sequence of steps instead of a single step. In a previous study [2], the authors suggested that a sequence of steps should consider 30 steps. So, in the IART data processing, the index $i$ is set equal to 30 (this number was derived from the estimation of a judge's field of view in real competition scenarios [9]). According to the IAAF rule, a sequence of steps is classified as 'legal' (mean LOGC $\mathrm{T}_{\mathrm{T}}$ is under the limit of human eye (LHE), with LHE fixed to $40 \mathrm{~ms}$ ) or 'illegal' (mean LOGC different colors (green for legal and red for illegal) are chosen for step sequence (SS) classification.

The second part of the flow chart is related to the coach operation mode [10]. This operational mode requires two input data from the user: the mean speed of the test and the athlete's height. Three parameters related to the performance, i.e., SL, SC, and the smoothness (S), and two related to the infringement, i.e., LOGC $_{\mathrm{T}}$ and LOGC (percentage of illegal step in a sequence), are assessed. Five biomechanical indices $(\mu, Q, \gamma, \alpha, \delta)$ related to the previous five parameters, customized for elite race walkers, are calculated. Finally, based on these five indices, a radar chart representation (with the assessment of the parameter $\varepsilon$ that represents the radar chart area) is derived to allow a simple graphical evaluation of the performance and infringement status of the athlete.

\subsection{Athletes and experimental Protocol}

Nine world-class Olympic race walkers (seven men and two women), members of three different national teams, took part in the experimental field tests. They were specialists of $20 \mathrm{~km}$ (three men and two women) and $50 \mathrm{~km}$ (four men). The participants gave an informed consent before the experimental sessions. The participants stature was within the reference population stature distribution (related to male and female Olympic race walkers). Indeed, they covered a large range from the 8th to the 97th percentile of these population [10]. Before the trials, all race walkers carried out a customized warm up of $15 \mathrm{~min}$ (including mobility exercises). An inertial sensor (measurement unit, according to the optimal concept) was located at the end of the athletes' vertebral column in correspondence of the L5-S1 inter-vertebral space. The standard experimental protocol consisted of 4 trials of $300 \mathrm{~m}$ race walking each, at different incremental mean speeds $(12.0 \mathrm{~km} / \mathrm{h}, 13.0 \mathrm{~km} / \mathrm{h}$, $14.0 \mathrm{~km} / \mathrm{h}$, and $14.5 \mathrm{~km} / \mathrm{h}$ ). In addition, in order to collect data even during high-speed race walking, two race walkers performed an additional test at the speed of $15.5 \mathrm{~km} / \mathrm{h}( \pm 0.1 \mathrm{~km} / \mathrm{h})$. The test leader followed the athletes on a bicycle during the test to collect LOGC high-speed data in a video as a benchmark for IART system evaluation. 


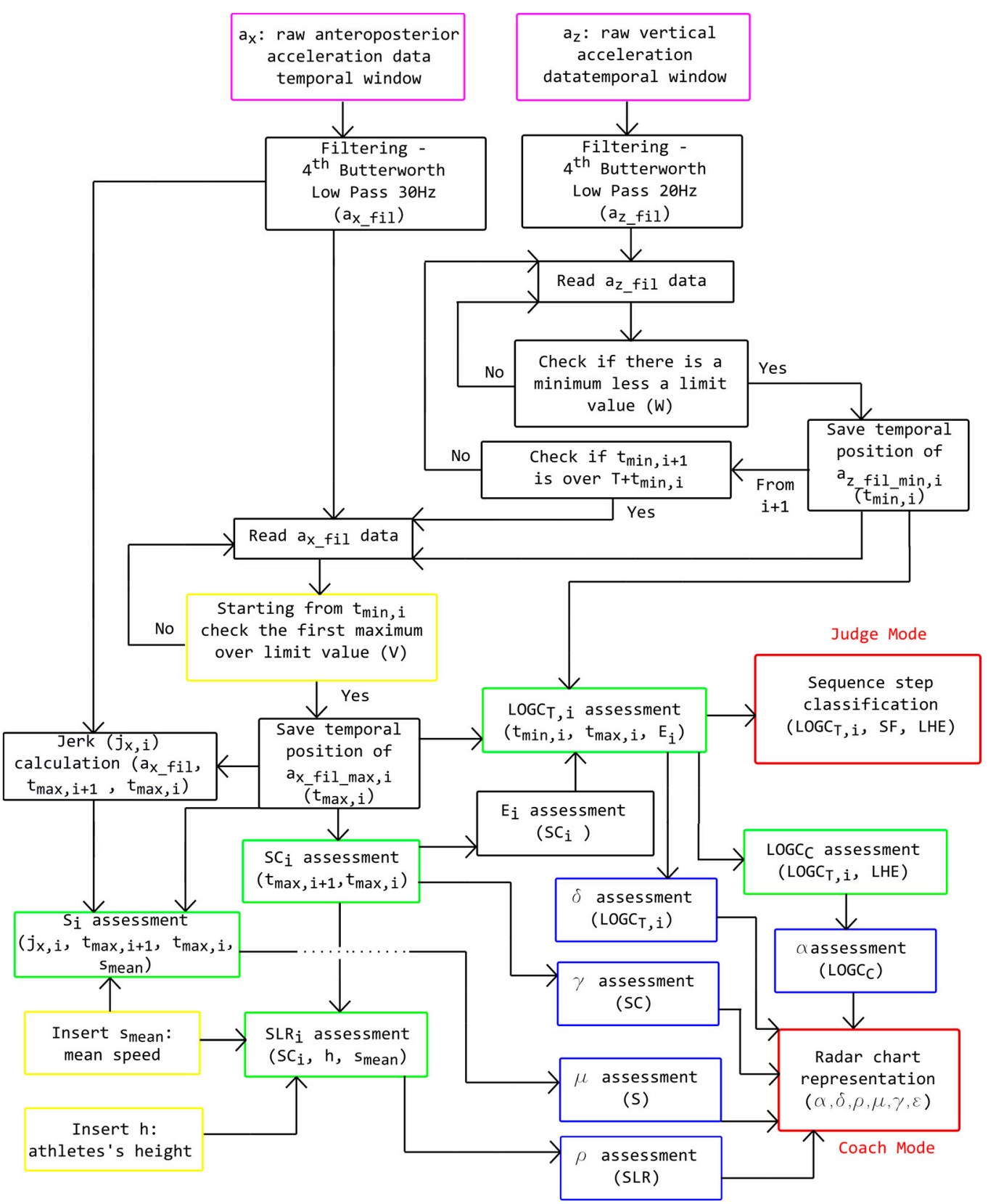

Figure 2. Flowchart of the process implemented in the management unit to obtain the output required (red box) for the two-mode operation. Raw data collected by the measurement unit are depicted in the violet box on the top. Performance and infringement parameters are located in the green box, and the related customized biomechanical indices are in the blue box. In the yellow boxes, one in the center and the other two at the bottom left, there are the additional data that have to be inserted by the user in the "coach" operation mode. LOGCт: loss of ground contact timing, LOGCc: percentage of illegal step in a sequence, SC: step cadence, S: smoothness, SLR: step length ratio $\mathrm{S}$ : smoothness related to the generic step $i, \mathrm{SC}_{\mathrm{i}}$ : smoothness related to the generic step $i$, LOGC $\mathrm{C}_{\mathrm{T}, \mathrm{i}}$ loss of ground contact timing related to the generic step $i$, SLRi: step length ratio related to the generic step $i, \alpha$ : biomechanical index related to $\mathrm{LOGC}_{\mathrm{T}}, \delta$ : biomechanical index related to LOGCC, $\mathrm{Q}$ : biomechanical index related to SLR, $\gamma$ : biomechanical index related to SC, $\mu$ : Q: biomechanical index related to S, SF: sample frequency, LHE: limit of human eye, $\mathrm{t}_{\text {max,i: }}$ time related to the maximum in the anteroposterior acceleration related to the generic step $i, t_{\min , i}$ time related to the maximum in the anteroposterior acceleration related to the generic step $i, \mathrm{~W}$ : fixed temporal value for the assessment of $t_{\min , \mathrm{i}} \mathrm{V}$ : fixed temporal value for the

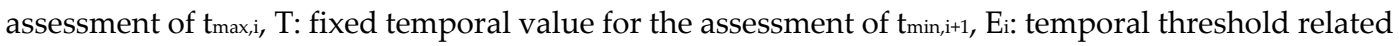
to the generic step $i$, jx,i jerk related to the generic step $i$, ax_fil: filtered anteroposterior acceleration; $\mathrm{az}_{-}$fil: filtered vertical acceleration; $\mathrm{az}_{-}$fil_min,i: minimum related to the generic step $i$ in $\mathrm{az}_{-}$fil, $\mathrm{ax}_{-}$fil_max,i: maximum related to the generic step $i$ in ax_fil. 


\section{Results}

\subsection{Judges Mode}

Starting from the collected data, we chose nine tests of three different athletes (two males, one specialized in $20 \mathrm{~km}$, and one in $50 \mathrm{~km}$, and one female specialized in $20 \mathrm{~km}$ ). The trials were chosen: (i) to cover five speeds of an elite race walker, from $12.0 \mathrm{~km}$ to $15.5 \mathrm{~km} / \mathrm{h}$; (ii) to analyze specialists of the main competitions. We choose five different speeds in order to obtain a good overview of the response of the proposed approach in relation to athletes' range of speeds. For each race walking test, excluding the initial acceleration phase of the athlete $(10 \mathrm{~s}), 180$ consecutive steps were considered related to six sequences of steps. A total amount of 54 sequences of steps (1620 athlete's steps) were evaluated. Starting from the LOGC used the binary classification method (see Section 2.2). Assuming as "true" the high-speed camera classification, false alarm rate (legal SS classified as illegal), miss alarm rate (illegal SS classified as legal), TPR (True Positive Rate: proportion of real legal SS which were correctly predicted as legal), FPR (False Positive Rate: proportion of real illegal SS which are predicted legal,) and accuracy were obtained (Table 1).

Table 1. LOGC data collected and statistics at different speeds. TPR: True Positive Rate, FPR: False positive Rate.

\begin{tabular}{llllll}
\hline & $\mathbf{1 2 . 0} \mathbf{~ k m} / \mathbf{h}$ & $\mathbf{1 3 . 0} \mathbf{~ k m} / \mathbf{h}$ & $\mathbf{1 4 . 0} \mathbf{~ k m} / \mathbf{h}$ & $\mathbf{1 4 . 5} \mathbf{~ k m} / \mathbf{h}$ & $\mathbf{1 5 . 5} \mathbf{~ k m} / \mathbf{h}$ \\
\hline LOGCT [ms] & $20 \pm 10$ & $35 \pm 10$ & $40 \pm 10$ & $45 \pm 10$ & $65 \pm 10$ \\
False Alarm [\%] & 8 & 24 & 33 & 14 & 100 \\
Miss Alarm [\%] & 64 & 52 & 22 & 55 & 0 \\
TPR [\%] & 92 & 76 & 66 & 86 & 0 \\
FPR [\%] & 64 & 52 & 22 & 54 & 0 \\
Accuracy [\%] & 91 & 73 & 70 & 79 & 94 \\
\hline
\end{tabular}

\subsection{Coach Mode}

We analyzed the overall data collected by nine race walkers related to four speeds between $12.0 \mathrm{~km}$ and $14.5 \mathrm{~km}$ (864 sequences of step, 25,920 steps). To give a practical representation of the output, in Figure 3, we report the biomechanical indices collected on radars charts, reported for two athletes at four different speeds.
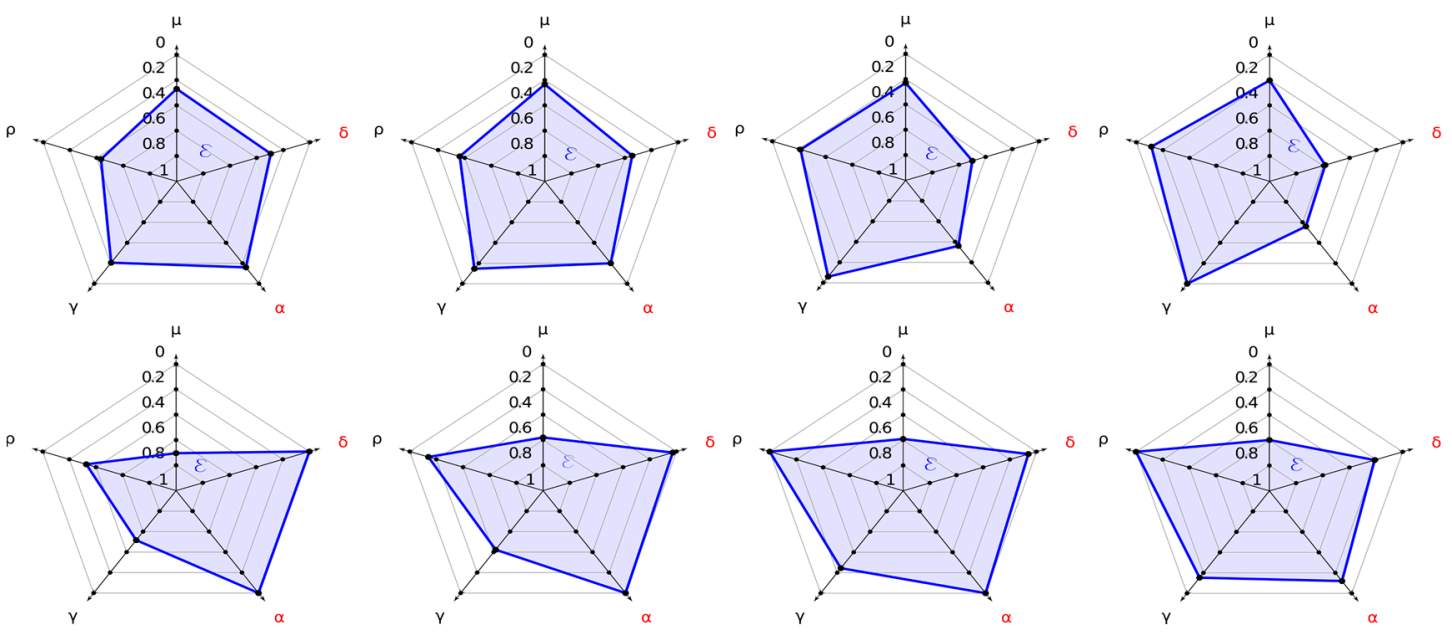

Figure 3. On the top, four radar charts for athlete 1 at increasing speeds (from left to right, $12.0 \mathrm{~km} / \mathrm{h}$, $13.0 \mathrm{~km} / \mathrm{h}, 14.0 \mathrm{~km} / \mathrm{h}$, and $14.5 \mathrm{~km} / \mathrm{h}$ ). On the bottom, four radar charts for athlete 2 at the same speeds. 


\section{Discussion}

To better understand the values reported in Table 1, we considered a previous study [11] reporting outdoor experimental tests that compared judges' assessment with respect to a camera evaluation (considered as benchmark). Analyzing these data with our binary method of classification for a sequence of step, the judges achieved the mean accuracy rate of $65 \%$. Although the results on speed analysis showed a decreasing trend of accuracy at speeds between $13.0 \mathrm{~km} / \mathrm{h}$ and $14.0 \mathrm{~km} / \mathrm{h}$, at these speeds the value of LOGC (see Table 1) was between 40 and $45 \mathrm{~ms}$ (around the LHE, where the discrimination between legal and illegal SS is more difficult), and a recent study [12] underlined that LOGC $\mathrm{T}_{\mathrm{T}}$ between $40 \mathrm{~ms}$ and $45 \mathrm{~ms}$ were usually detected by no more than $37 \%$ of judges (IART in these conditions guarantee accuracy over $70 \%$ ). In addition, according to the same study [12], for LOGC timing under $33 \mathrm{~ms}$ (typical for example of speeds under $13.0 \mathrm{~km}$ ), $12.5 \%$ of judges detected a non-visible LOGC (that, according to the LHE, can be considered as False Alarm cases), whereas IART showed a better performance (False Alarm equal to $8 \%$ ). Finally, also for elevated speed $(15.5 \mathrm{~km} / \mathrm{h}$, for which the LOGC $\mathrm{T}$ is over $50 \mathrm{~ms}$ ), IART accuracy reached the value of $94 \%$ (also thanks to the higher LOGC $_{\mathrm{T}}$ that helped the discrimination between legal and illegal SS), a performance better than judges' performance for LOGC ${ }_{\text {т }}$ over $60 \mathrm{~ms}$ (85\% of accuracy) [12]. From the performance analysis point of view, the radar chart representation allows to understand strong and critical points that characterize an athlete's technique. In Figure 3, the radar charts sequences evaluated for different speeds underline how Athlete 2 had step length values (@) higher than the step cadence values $(\gamma)$; therefore, the step length represents the strength technical point of athlete's gesture. In contrast, Athletes 1 had the strongest technical feature in step cadence. Finally, $\varepsilon$ can suggest the speeds that would allow achieving, at the same time, optimal values of Step Length Ratio (SLR, step length normalized to the athlete's height) and SC, while ensuring an acceptable level of correct technique (13 and $14 \mathrm{~km} / \mathrm{h}$ in Figure 3). This assessment is compatible with previous results [12] which indicate the values of $14 \mathrm{~km} / \mathrm{h}$ for men and $13 \mathrm{~km} / \mathrm{h}$ for women as the speed limits for avoiding visible loss of contact.

\section{Conclusions}

In this paper, we described how the wearable inertial system IART can be used to help end users of race walking applications for the assessment of athletes' performances and infringements. The results of the experimental validation, in a real training scenario with world-class Olympic race walkers, confirmed that: (i) the "judge" mode could ensure better accuracy then an actual judge's evaluation for step sequence classification; (ii) the "coach" mode through the radar chart offers the possibility to build a customized profile of a race walker, useful for training improvement.

Acknowledgments: We would like to thank all athletes, coaches, and experts for their participation and precious support in the experimental tests.

Conflicts of Interest: The authors declare no conflict of interest.

\section{References}

1. IAAF. Race Walking a Guide to Judging and Organising. A Guide for Judges, Officials, Coaches and Athletes; World Athletics, Monaco, 2018.

2. Di Gironimo, G.; Caporaso, T.; Amodeo, G.; Del Giudice, D.M.; Lanzotti, A.; Odenwald, S. Outdoor tests for the validation of an inertial system able to detect illegal steps in race-walking. Procedia Eng. 2016, 147, $544-549$.

3. Di Gironimo, G.; Caporaso, T.; Del Giudice, D.M.; Tarallo, A.; Lanzotti, A. Development of a new experimental protocol for analysing the race-walking technique based on kinematic and dynamic parameters. Procedia Eng. 2016, 147, 741-746.

4. "Olympic.org": $50 \mathrm{~km}$ walk men. Available online: https://www.olympic.org/rio-2016/athletics/50kmwalk-men (accessed on 20 May 2020).

5. Hanley, B. A biomechanical analysis of world-class senior and junior race walkers. New Stud. Athl. 2013, $28,75-82$. 
6. Lee, J.B.; Mellifont, R.B.; Burkett, B.J.; James, D.A. Detection of illegal race walking: A tool to assist coaching and judging. Sensors 2013, 13, 16065-16074.

7. Taborri, J.; Palermo, E.; Rossi, S. Automatic Detection of Faults in Race Walking: A Comparative Analysis of Machine-Learning Algorithms Fed with Inertial Sensor Data. Sensors 2019, 19, 1461.

8. Di Gironimo, G.; Caporaso, T.; Del Giudice, D.M.; Lanzotti, A. Towards a new monitoring system to detect illegal steps in race-walking. IJIDeM 2017, 11, 317-329.

9. Caporaso, T.; Grazioso, S. The IART system: Inertial Assistant Referee and Trainer for race-walking. Sensors 2020, 20, 783.

10. Caporaso, T.; Grazioso, S.; Di Gironimo, G.; Lanzotti, A. Biomechanical indices represented on radar chart for assessment of performance and infringements in elite race walkers. Sports Eng. 2020, 23, 4, doi:10.1007/s12283-019-0317-2.

11. Knicker, A.; Loch, M. Race walking technique and judging-The final report of the International Athletic Foundation research project. New Stud. Athl. 1990, 5, 25-38.

12. Hanley, B.; Tucker, C.B.; Bissas, A. Assessment of IAAF racewalk judges' ability to detect legal and nonlegal technique. Front. Sports Act. Living 2019, 1, 9.

(C) 2020 by the authors. Licensee MDPI, Basel, Switzerland. This article is an open access article distributed under the terms and conditions of the Creative Commons Attribution (CC BY) license (http://creativecommons.org/licenses/by/4.0/). 